\title{
Do Clinical Features and Survival of Single Hormone Receptor Positive Breast Cancers Differ from Double Hormone Receptor Positive Breast Cancers?
}

\author{
Char-Hong Ng${ }^{1}$, Nirmala Bhoo Pathy ${ }^{2}$, Nur Aishah Taib ${ }^{1}$, Gwo-Fuang Ho ${ }^{3}$, Kein- \\ Seong Mun ${ }^{4}$, Anthony Rhodes ${ }^{4}$, Lai-Meng Looi ${ }^{4}$, Cheng-Har Yip ${ }^{1 *}$
}

\begin{abstract}
The significance of the single hormone receptor positive phenotype of breast cancer is still poorly understood. The use of hormone therapy has been found to be less effective for this type, which has a survival outcome midway between double positive and double negative phenotypes. The aim of this study was to investigate differences in patient and tumor characteristics and survival between double-receptor positive (ER+PR+), double receptor negative (ER-PR-) and single receptor positive (ER+PR- and ER-PR+) breast cancer in an Asian setting. A total of 1,992 patients with newly diagnosed stage I to IV breast cancer between 2003 and 2008 , and where information on ER and PR were available, were included in this study. The majority of patients had ER+PR+ tumors (n=903: 45.3\%), followed by 741 (37.2\%) ER-PR-, 247 (12.4\%) ER+PR-, and 101 (5.1\%) ER-PR+tumors. Using multivariate analysis, ER+PR- tumors were 2.4 times more likely to be grade 3 compared to ER+PR+ tumors. ER+PR- and ER-PR+ tumors were $82 \%$ and $86 \%$ respectively less likely to be grade 3 compared with ER-PR- tumors. ER-PR+ tumours were associated with younger age. There were no survival differences between patients with ER+PR+ and ER-PR+ tumors. However, ER+PR- tumors have poorer survival compared with ER+PR+ tumours. ER-PR- tumours had the worst survival. Adjuvant hormonal therapy with tamoxifen was found to have identical survival advantage in patients with ER+PR+ and ER-PR+ tumors whereas impact was slightly lower in patients with ER+PR- tumors. In conclusion, we found ER+PR- tumors to be more aggressive and have poorer survival when compared to $\mathrm{ER}+\mathrm{PR}+$ tumors, while patients with $\mathrm{ER}-\mathrm{PR}+$ tumours were younger, but had a similar survival to their counterparts with $E R+P R+$ tumours.
\end{abstract}

Keywords: Breast cancer - hormonal receptors - ER-PR+ subtype - survival outcome

Asian Pac J Cancer Prev, 15 (18), 7959-7964

\section{Introduction}

The steroid hormone receptors, estrogen receptors (ER) and progesterone receptors (PR) are important prognostic markers in the management of breast cancer and in addition they predict response to endocrine therapy. The double positive phenotype (ER+PR+) is known to be associated with older women, smaller tumors, lower grade and have better prognosis and good response to hormonal therapy as compared to the double negative phenotype. However, the significance of the single hormone receptor positive phenotype, which includes the ER+PR- and ER$\mathrm{PR}+$ tumors, is still poorly understood. The proportion of ER+PR- tumors has been reported to be $12-17 \%$ (Dunnwald et al., 2007; Rakha et al., 2007; Rakha et al., 2010) while the proportion of ER-PR+ tumors is much less, reported to be 1-10\% (Rakha et al., 2007; Yu et al., 2008a; Rakha et al., 2010). Hormone therapy has been found to be less effective in the single hormone receptor positive phenotype and they have a survival outcome between double-positive and double-negative phenotypes (Bardou et al., 2003; Rakha et al., 2007). Several studies have found that up to $10 \%$ of estrogen receptor-negative (ER-) breast cancers are progesterone receptor-positive (PR+) (Rakha et al., 2010) although recent evidence shows that the percentage is much lower when more sensitive immunohistochemical (IHC) methods for ER determination are used (Rakha et al., 2007; Rhodes and Jasani, 2009; Rakha et al., 2010). Cold ischaemia time, that is, the interval between surgical removal of the breast and fixation in formalin, leads to degradation of the labile ER and PR receptors, and the ER may be more labile than PR, and hence could degrade more readily than PR. Hence, a proportion of ER-PR+ tumors may be actually be ER+PR+ 
because of delayed tissue fixation or technical failure of the IHC assay (Rhodes et al., 2000; Nadji et al., 2005; Jasani et al., 2006; De Maeyer et al., 2008; Nadji, 2008). If this is true, then it would be expected that the ER-PR+ phenotype would have similar tumor characteristics to the $\mathrm{ER}+\mathrm{PR}+$ phenotype. However, more recent studies have shown that ER-PR+ tumors exhibit more aggressive characteristics than double-hormone receptor-positive cancers (Rakha et al., 2007; Rakha et al., 2010), are significantly associated with younger age(Rhodes and Jasani, 2009) and have survival outcome between double positive and double negative phenotypes(Rakha et al., 2007). This has led to the question whether the ER-PR+ subtype could be a true biological entity or a technical artifact.

The aim of this study was to investigate the difference in patient and tumor characteristics and survival between double-receptor positive $(\mathrm{ER}+\mathrm{PR}+)$, double receptor negative (ER-PR-) and single receptor positive (ER+PRand ER-PR+) breast cancer in an Asian setting.

\section{Materials and Methods}

\section{Study population}

Data from the University Malaya Medical Centre (UMMC) Breast Cancer Registry was used in this study. UMMC is an academic tertiary hospital situated in the relatively affluent part of Kuala Lumpur (Malaysia). Its breast cancer registry comprise a prospective database of 3795 consecutive women who were newly diagnosed with breast cancer between 1993 and 2008, and had received approval from the institutional review board.

As testing for both hormone receptors was only routinely carried out since the early 2000s in UMMC, 1992 patients with newly diagnosed stage I to IV breast cancer between 2003 and 2008, and where information on ER and PR were available, were included in this study.

\section{Immunohistochemistry}

The estrogen and progesterone receptor status of the cases was determined by IHC, using the ER clone SP1 (Neomarkers, USA ) and PR clone PgR636 (DAKO, Denmark), respectively. Briefly, 4-micron-thick microtomed sections were de-paraffinised and endogenous peroxidase blocked in $0.3 \%$ hydrogen peroxide. Antigen retrieval was achieved in TRIS EDTA buffer (pH9.0) at $990 \mathrm{C}$ in a $750 \mathrm{~W}$ Microwave oven for 20 minutes. The slides were then incubated in the respective primary antibodies (ER at 1:100 dilution and PR at 1:200 dilution) for 30 minutes at room temperature $\left(24^{\circ} \mathrm{C}\right)$. Detection was achieved using an avidin-biotin based system and horseradish peroxidase label (Dako, Denmark) and visualization achieved by the addition of an hydrogen peroxide substrate and diaminobenzidene chromogen (Dako, Denmark). The tumor was deemed ER or PR positive when $10 \%$ or more of the invasive tumor nuclei were stained for the respective antibody, regardless of staining intensity.

Analysis of hormonal receptor status with clinicopathological variables

Patients were divided into four categories namely $\mathrm{ER}+\mathrm{PR}+, \mathrm{ER}+\mathrm{PR}+, \mathrm{ER}-\mathrm{PR}+$ and ER-PR-. Variables studied in relation to these four groups were age at diagnosis, ethnicity (Chinese, Malay, Indian), tumor size (available as largest dimension in millimeters), lymph node involvement (available as number of tumor-positive nodes and categorized into yes, no, unknown), tumor grade (Modified Bloom-Richardson classification; grade 1 , grade 2 , grade 3 , unknown), histological type (invasive ductal, invasive lobular, others), presence of distant metastasis (yes, no, unknown) and treatment modalities (surgery, radiotherapy, chemotherapy, hormone therapy).

Data on mortality were obtained from the hospital medical records, as well as active follow-up through the next-of-kin of patients. In addition, vital status was verified through direct linkage with the National Registration Department in Malaysia. In this hospital based cancer registry, information on cause of death or cancer recurrence was not available for the majority of patients.

\section{Data analysis}

Categorical variables were compared using the chi-square test and continuous variables using one way analysis of variance (ANOVA). Univariable and multivariable logistic regression analyses were used to determine the association between patient and tumor characteristics (independent) and hormone receptor status (dependent).

Overall survival was estimated using Kaplan-Meier analyses and compared by log-rank test. Cox regression analysis was performed to estimate the relative risk for all-cause mortality expressed as hazard ratio (HR) between women of different hormone receptor status. Time at entry was date of diagnosis with breast cancer, and exit time was date of death (from any cause), date at last contact, or November 2011 (linkage with national mortality registry in Malaysia). All analyses were performed using SPSS version 16.0 (SPSS Inc, Chicago, USA).

\section{Results}

The majority of patients had ER+PR+ tumors ( $\mathrm{n}=903$ : $45.3 \%$ ), followed by 741 (37.2\%) ER-PR-, 247 (12.4\%) ER+PR-, and $101(5.1 \%)$ ER-PR+ tumors. Mean age at diagnosis of overall patients was 53 years. Median tumor size at presentation was $30 \mathrm{~mm}$. The distribution of tumor characteristics by hormone receptor status is presented in Table 1.

Patients with ER+PR-phenotypes were older although not significantly than ER+PR+ (OR:1.01; 95\%CI: 1.00 1.02) and ER-PR- phenotypes (OR:1.02; 95\%CI: 1.011.03) (Table 2a). ER+PR- phenotypes were associated with larger tumor when compared with ER+PR+ (OR:1.06; 95\%CI: 1.02-1.10) and smaller when compared to ER-PR- phenotypes (OR:0.97; 95\%CI: 0.93-1.00). ER+PR- phenotypes were 2.73 times more likely to be Grade 3 tumors, compared to ER+PR+ cases, (OR:2.73; 95\%CI: 1.51-4.95) (Table 2) and 85\% less likely to be associated with a grade 3 tumor compared to the ER-PRphenotypes (OR: 0.15; 95\%CI:0.07-0.33).

Patients with ER-PR+ phenotypes were significantly younger than ER+PR+ (OR:0.96; 95\%CI: 0.94-0.98) and ER-PR- phenotypes (OR:0.97; 95\%CI: 0.96-0.99) (Table 
Comparison of Single Hormone Receptor Positive and Double Hormone Receptor Positive Breast Cancers

2b). ER-PR- phenotype was associated with a smaller tumor when compared to ER-PR- phenotype (OR:0.93; 95\%CI: 0.87-0.99). ER-PR+ phenotypes were 2.5 times more likely to be Grade 3 tumors, compared to ER+PR+ cases, (OR:2.50; 95\%CI: 1.11-5.63) and 86\% less likely to be associated with a grade 3 tumor compared to the ER-PR- phenotypes (OR: 0.14; 95\%CI:0.05-0.36).
Compared to ER+PR+ tumors, only grade 3 (OR:2.40; 95\% CI: 1.31-4.40) remained significantly associated with ER+PR- phenotypes following multivariable adjustment. When compared to ER-PR-phenotypes, ER+PR- tumors were less likely to be associated with higher tumor grade (Table 2a).

Compared to $\mathrm{ER}+\mathrm{PR}+$ tumors, only younger age

Table 1. Distribution of Characteristics by Hormone Receptor Status in 1992 Women with Breast Cancer

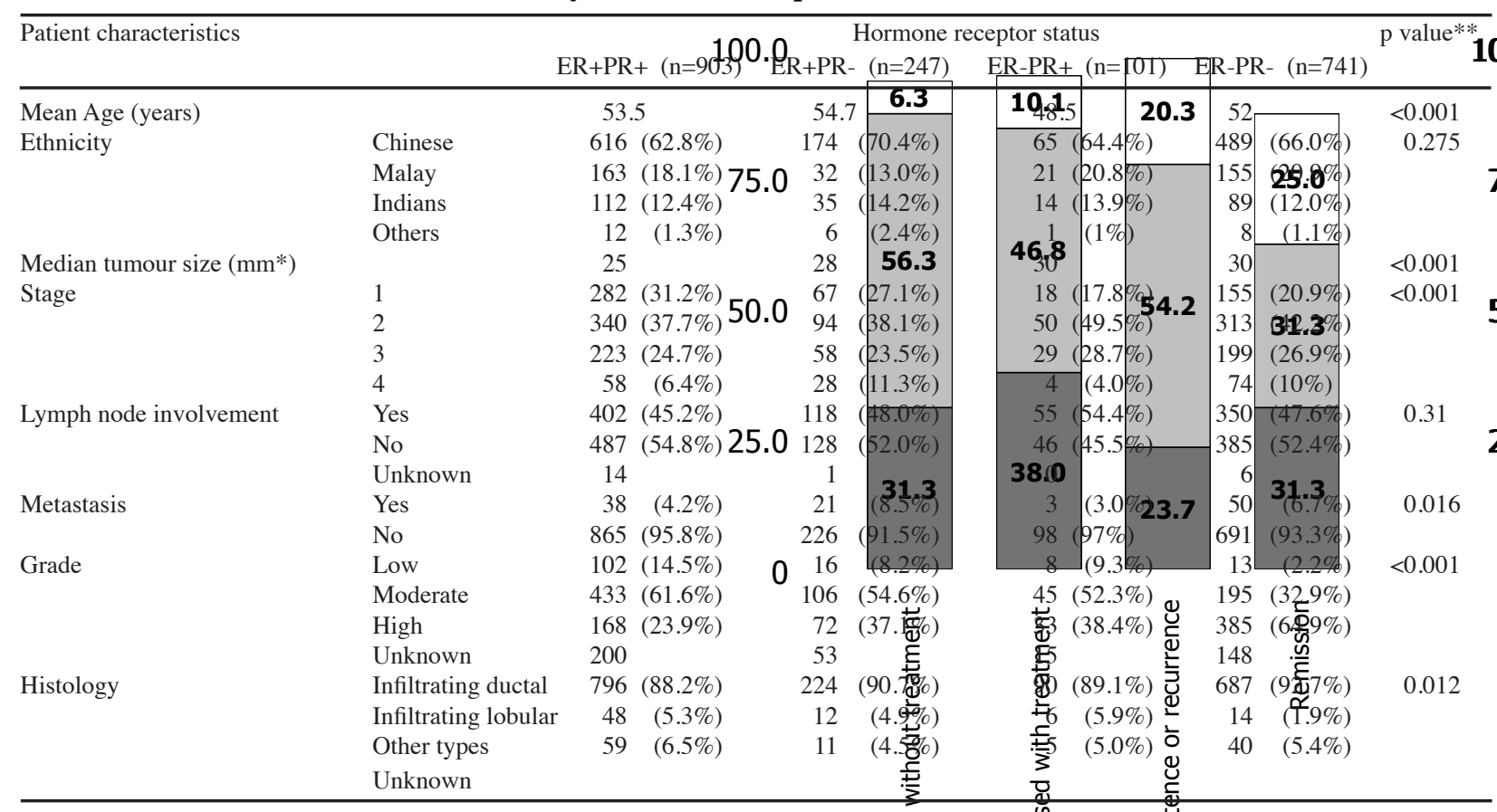

*Available in 1938 patients; **Derived using one-way Annova for age, Kruskal Wallis for tumour size, and Chi Square test for categorical variables

Table 2a. Association between Patient/tumour Charactersitics and Hormone Receptor Status

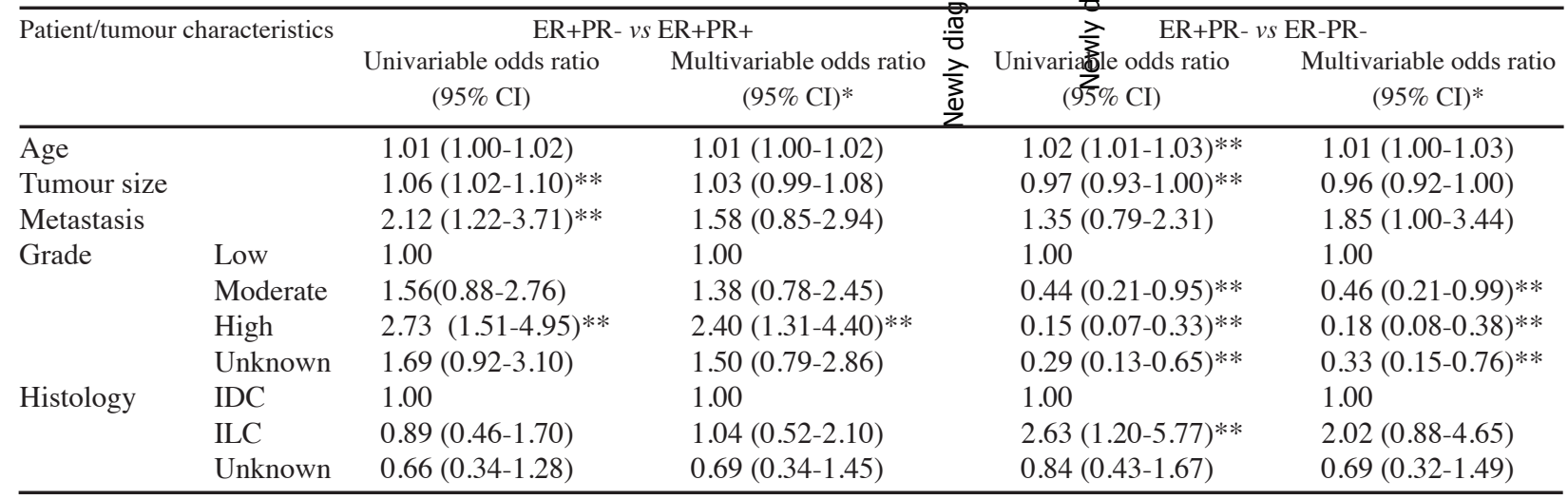

*Derived using logistic regression model adjusted mutually for age, tumour size, metastasis, grade and histology; IDC: infiltrating ductal carcinoma; ILC: infiltrating lobular carcinoma; **Statistically significant

Table 2b. Association between Patient/tumour Characteristics and Hormone Receptor Status



*Derived using logistic regression model adjusted mutually for age, tumour size, metastasis, grade and histology; IDC: infiltrating ductal carcinoma; ILC: infiltrating lobular carcinoma; **Statistically significant 
Table 3. Pattern of Treatment by Hormone Receptor Status

\begin{tabular}{|c|c|c|c|c|c|c|}
\hline \multirow{2}{*}{\multicolumn{2}{|c|}{ Treatment }} & \multicolumn{4}{|c|}{ Hormone receptor status } & \multirow[t]{2}{*}{ p value * } \\
\hline & & $\mathrm{ER}+\mathrm{PR}+(\mathrm{n}=903)$ & $\mathrm{ER}+\mathrm{PR}-(\mathrm{n}=247)$ & ER-PR+ $(n=101)$ & ER-PR- $(n=741)$ & \\
\hline \multirow[t]{3}{*}{ Surgery } & No & $51 \quad(5.6 \%)$ & $14 \quad(4.9 \%)$ & $3 \quad(3.0 \%)$ & $49 \quad(5.9 \%)$ & 0.661 \\
\hline & Yes & $844(94.4 \%)$ & $232(95.1 \%)$ & 97 (97\%) & $687(94.1 \%)$ & \\
\hline & Unknown & $8 \quad 1$ & 15 & & & \\
\hline \multirow[t]{3}{*}{ Radiotherapy } & No & $307(39.7 \%)$ & $86(40.2 \%)$ & $22 \quad(28.9 \%)$ & $240(38.3 \%)$ & 0.309 \\
\hline & Yes & $467(60.3 \%)$ & $128(59.8 \%)$ & $54(71.1 \%)$ & $387(61.7 \%)$ & \\
\hline & Unknown & $129 \quad 33$ & 25114 & & & \\
\hline \multirow[t]{3}{*}{ Chemotherapy } & No & $275(34.3 \%)$ & $61(26.4 \%)$ & $12(12.9 \%)$ & $136(19.5 \%)$ & $<0.001$ \\
\hline & Yes & $526(65.7 \%)$ & $170(73.6 \%)$ & $81 \quad(87.1 \%)$ & $563(80.5 \%)$ & \\
\hline & Unknown & $102 \quad 16$ & 842 & & & \\
\hline \multirow[t]{3}{*}{ Hormone therapy } & No & $34 \quad(4.0 \%)$ & $11 \quad(4.6 \%)$ & $13(14.0 \%)$ & $524(95.4 \%)$ & $<0.001$ \\
\hline & Yes & $817(96.0 \%)$ & $226(95.4 \%)$ & $80 \quad(86.0 \%)$ & $25 \quad(4.6 \%)$ & \\
\hline & Unknown & $52 \quad 10$ & 8192 & & & \\
\hline
\end{tabular}

*Derived using Chi Square test and does not include patients with unknown treatment

Table 4. Hormone Receptor Status and Overall Mortality Risk Following

\begin{tabular}{lcc}
\hline $\begin{array}{l}\text { Hormone receptor } \\
\text { status }\end{array}$ & $\begin{array}{c}\text { Hazard ratio } \\
\text { (95\% confidence interval) }\end{array}$ & $\begin{array}{c}\text { Adjusted hazard ratio } \\
(95 \% \text { confidence interval)* }\end{array}$ \\
\hline ER+PR+ & 1.00 & 1.00 \\
ER+PR- & $1.28(0.92-1.79)$ & $1.34(0.95-1.89)$ \\
ER-PR+ & $1.03(0.63-1.68)$ & $1.05(0.64-1.75)$ \\
ER-PR- & $2.31(0.63-1.68)$ & $2.49(1.97-3.17)$ \\
\hline
\end{tabular}

*Derived using Cox regression adjusted for age, ethnicity, tumour size, lymph node status, metastasis, tumour grade

Table 5. Impact of Hormone Treatment on Mortality following Breast cancer by ER/PR Status

\begin{tabular}{lcc}
\hline $\begin{array}{l}\text { Hormone receptor } \\
\text { status }\end{array}$ & \multicolumn{2}{c}{ Hormone Therapy } \\
& $\begin{array}{c}\text { No } \\
\text { Yes }\end{array}$ \\
\hline ER+PR+ & 1.00 & $0.18(0.11-0.30)^{*}$ \\
ER+PR- & 1.00 & $0.30(0.12-0.76)^{*}$ \\
ER-PR+ & 1.00 & $0.19(0.06-0.58)^{*}$ \\
ER-PR- & 1.00 & $0.63(0.26-1.54)$ \\
*Statistically significant & &
\end{tabular}

(OR:0.96; 95\%CI: 0.94-0.98) remained significantly associated with ER-PR+ phenotypes following multivariable adjustment. When compared to ER-PRphenotypes, ER-PR+ tumors were also more likely to be associated with invasive lobular histology than invasive ductal histology (adjusted OR: 3.36; 95\%CI: 1.14-9.96), and less likely to be associated with higher tumor grade following multivariable adjustment.

There were no differences in patterns of surgery or radiotherapy administration between the different categories of hormone receptor status. Chemotherapy was most likely administered to patients with ER-PR+ tumors $(87.1 \%)$, followed by ER-PR- (80.5\%), ER+PR$(73.6 \%)$, and $\mathrm{ER}+\mathrm{PR}+(65.7 \%) ; \mathrm{p}<0.001$. Hormone therapy administration was highest in ER+PR+ tumors (96.0\%), followed by ER+PR- (95.4\%), and ER-PR+ $(86.0 \%)$ phenotypes (Table 3). There were no survival differences between patients with $\mathrm{ER}+\mathrm{PR}+$ tumors and ER-PR+ tumors. However, those with the ER+PR-, and ER-PR- phenotypes had significantly poorer survival where ER-PR- phenotypes had the worst survival (Table 4).

We additionally looked at the impact of hormone therapy in patients with ER+ or PR+ tumors. Adjuvant hormonal therapy with tamoxifen was found to have identical survival advantage in patients with $\mathrm{ER}+\mathrm{PR}+$ and ER-PR+ tumors whereas impact was slightly lower in patients with ER+PR- tumors (Table 5).

\section{Discussion}

In this Asian series of breast cancers, patients with the ER-PR+ phenotype although significantly associated with a younger age at diagnosis compared to patients with the other phenotypes, have tumors with no distinct histopathological characteristics compared to $\mathrm{ER}+\mathrm{PR}+$ and ER-PR- tumors and no overall survival differences and mortality impact following hormonal therapy.

We found a lower proportion of double HR+ (45.3\%) and higher proportion of double HR- (37.2\%) tumors when compared to the West (Rhodes et al., 2000; Pathy et al., 2011) which is consistent with other Asian studies (Chow and Ho, 2000; Yip, 2009). Western studies have shown that the percentages of $\mathrm{ER}+\mathrm{PR}+$ were in the region of $55-65 \%$ and ER-PR- were $18-25 \%$. This is probably due to the fact that compared to Caucasian women, Asian patients are relatively younger (below 50 years) at diagnosis (Yip, 2009) and also use less hormone replacement therapy (Pathy et al., 2011). It has been previously shown that hormone replacement therapy use is associated with higher rates of ER positive breast cancers (Hwang et al., 2005).

In this study, in univariable analysis, ER-PR+ cancers were associated with a younger age and a higher grade, when compared to ER+PR+cancers, but with multivariable analysis, only younger age remained associated with ER$\mathrm{PR}+$ cancers when compared to ER+PR+ cancers. There was no significant association with larger tumors or higher grade, as seen with other studies. (Rakha et al.; Dunnwald et al., 2007). The younger age at diagnosis suggests that ER-PR+ breast cancer may be a separate biological entity; if this phenotype is due to a technical artifact, then the rate of ER-PR+ breast cancer would be constant through all the different age groups. When we compare ER-PR+ breast cancer with the double negative subgroup, the ERPR- subgroup were more likely to have higher grade, and less likely to be infiltrating lobular carcinoma compared with the ER-PR+ subgroup. Hence comparing ER-PR+ with the double negatives and double positives, ER-PR+ cancers appear to be more like $\mathrm{ER}+\mathrm{PR}+$ breast cancer except for a younger age.

Double HR+ has been shown to have good response 
to hormonal therapy and survival in comparison to double HR- where the reported response rate is negligible (Rakha et al., 2007; Rakha et al., 2010). However, the significance of the single $\mathrm{HR}+$ phenotype that includes $\mathrm{ER}+\mathrm{PR}$ - and ER-PR+ in relation to hormonal therapy and survival is still poorly understood. In previous studies, only ER status have been determined, and hormone therapy has been recommended predominantly on the ER positive status of patients, as there has been little evidence that tamoxifen is effective in ER negative patients. However, if ER alone is considered, then a small proportion of patients who are ER-PR+ (5.1\% in this study) will be denied tamoxifen. Dowsett et al showed that ER - patients showed a strong trend to benefit from tamoxifen, which was largely confined to the ER-PR+ group (Dowsett et al., 2006). Similarly in our study, the ER-PR+ patients derive a better overall survival when on tamoxifen compared to those who were not taking Tamoxifen. However this beneficial effect of tamoxifen was not seen in other studies, (Rakha et al., 2007; Yu et al., 2008b) which concluded ER-PR+ patients gain less benefit than patients with ER+PR+ tumors when they were on tamoxifen.

In ER+ patients who received adjuvant hormonal therapy, absence of PR was an independent predictor of development of recurrence and shorter survival and hence, a poor response to hormonal therapy. (Rakha et al., 2007) Similarly, other studies have shown that PR status is an independent predictive factor for hormonal adjuvant therapy (Wyss et al., 1992; Bardou et al., 2003; Yu et al., 2007).

In our study, we found that ER+PR+ and ER-PR+ tumors have no significant difference in overall survival, while there is a significant survival advantage when compared to ER+PR- and ER-PR- tumours. This finding contradicts the findings of Rakha et.al (Rakha et al., 2007) where, compared to double positive subgroup, ER+PRand ER-PR+ subgroups have poorer outcome. It should be emphasised that it can be difficult to ascertain the true clinical significance of the ER-PR+ phenotype, when analysing a retrospective series of cases. ( $\mathrm{Ng}$ et al., 2012) It only presents in a small number of cases (1-10\%) and indeed re-testing of cases by some authors utilising more sensitive immunohistochemical detection systems and lower cut points suggests that at least some of these cases are double receptor positive using alternative assessment criteria (Hammond et al.; Nadji et al., 2005; De Maeyer et al., 2008; Nadji, 2008). In addition hormone receptors are notoriously labile and unless tissues are fixed promptly and the cold ischaemia time between surgical removal and immersion in fixative is kept to a minimum, there is the confounding factor of receptor degradation and consequent false negative assay results (Khoury et al., 2009) All these factors need to be taken into consideration when considering the clinical relevance of the ER-PR+ result.

In conclusion, in this cohort of Malaysian women, apart from age differences, we did not find any histopathological difference between ER-PR+ and ER+PR+ tumors. There was also no survival difference observed between the above subgroups.

However, the ER-PR+ tumors exhibited less aggressive characteristics compared to ER-PR- tumors, and patients with the former had a higher overall survival than the latter. Although ER-PR+ appears to be a distinct biological subtype, there is no effect on overall survival and Tamoxifen has been shown to be of benefit in this ER-PR+ subtype.

\section{References}

Bardou VJ, Arpino G, Elledge RM, et al (2003). Progesterone receptor status significantly improves outcome prediction over estrogen receptor status alone for adjuvant endocrine therapy in two large breast cancer databases. J Clin Oncol, 21, 1973-9.

Chow LW, Ho P (2000). Hormonal receptor determination of 1,052 Chinese breast cancers. J Surg Oncol, 75, 172-5.

De Maeyer L, Van Limbergen E, De Nys K, et al (2008). Does estrogen receptor negative/progesterone receptor positive breast carcinoma exist? J Clin Oncol, 26, 335-6; author reply 6-8.

Dowsett M, Houghton J, Iden C, et al (2006). Benefit from adjuvant tamoxifen therapy in primary breast cancer patients according oestrogen receptor, progesterone receptor, EGF receptor and HER2 status. Ann Oncol, 17, 818-26.

Dunnwald LK, Rossing MA, Li CI (2007). Hormone receptor status, tumor characteristics, and prognosis: a prospective cohort of breast cancer patients. Breast Cancer Res, 9, 6 .

Hammond ME, Hayes DF, Dowsett M, et al American Society of Clinical Oncology/College Of American Pathologists guideline recommendations for immunohistochemical testing of estrogen and progesterone receptors in breast cancer. J Clin Oncol, 28, 2784-95.

Hwang ES, Chew T, Shiboski S, et al (2005). Risk factors for estrogen receptor-positive breast cancer. Arch Surg, 140, 58-62.

Jasani B, Douglas-Jones A, Rhodes A, et al (2006). Measurement of estrogen receptor status by immunocytochemistry in paraffin wax sections. Methods Mol Med, 120, 127-46.

Khoury T, Sait S, Hwang H, et al (2009). Delay to formalin fixation effect on breast biomarkers. Mod Pathol, 22, 1457-67.

Nadji M (2008). Quantitative immunohistochemistry of estrogen receptor in breast cancer: "much ado about nothing!". Appl Immunohistochem Mol Morphol, 16, 105-7.

Nadji M, Gomez-Fernandez C, Ganjei-Azar P, et al (2005). Immunohistochemistry of estrogen and progesterone receptors reconsidered: experience with 5,993 breast cancers. Am J Clin Pathol, 123, 21-7.

$\mathrm{Ng} \mathrm{CH}$, Pathy NB, Taib NA, et al (2012). The estrogen receptor negative-progesterone receptor positive breast carcinoma is a biological entity and not a technical artifact. Asian Pac J Cancer Prev, 13, 1111-3.

Pathy NB, Yip CH, Taib NA, et al (2011). Breast cancer in a multi-ethnic Asian setting: results from the SingaporeMalaysia hospital-based breast cancer registry. Breast, 20 , 75-80.

Rakha EA, El-Sayed ME, Green AR, et al (2007). Biologic and clinical characteristics of breast cancer with single hormone receptor positive phenotype. J Clin Oncol, 25, 4772-8.

Rakha EA, Reis-Filho JS, Ellis IO Combinatorial biomarker expression in breast cancer. Breast Cancer Res Treat, 120, 293-308.

Rakha EA, Reis-Filho JS, Ellis IO (2010). Combinatorial biomarker expression in breast cancer. Breast Cancer Res Treat, 120, 293-308.

Rhodes A, Jasani B (2009). The oestrogen receptor-negative/ 


\section{Char-Hong Ng et al}

progesterone receptor-positive breast tumour: a biological entity or a technical artefact? J Clin Pathol, 62, 95-6.

Rhodes A, Jasani B, Balaton AJ, et al (2000). Frequency of oestrogen and progesterone receptor positivity by immunohistochemical analysis in 7016 breast carcinomas: correlation with patient age, assay sensitivity, threshold value, and mammographic screening. J Clin Pathol, 53, 688-96.

Wyss P, Rageth JC, Unger C, et al (1992). [Prognostic and therapeutic significance of steroid receptors in invasive breast cancer]. Geburtshilfe und Frauenheilkunde, 52, 611-6.

Yip CH (2009). Breast cancer in Asia. Methods Mol Biol, 471, 51-64.

Yu KD, Di GH, Wu J, et al (2008a). Breast cancer patients with estrogen receptor-negative/progesterone receptor-positive tumors: being younger and getting less benefit from adjuvant tamoxifen treatment. J Cancer Res Clin Oncol, 134, 1347-54.

Yu KD, Di GH, Wu J, et al (2008b). Breast cancer patients with estrogen receptor-negative/progesterone receptor-positive tumors: being younger and getting less benefit from adjuvant tamoxifen treatment. J Cancer Res Clin Oncol, 134, 1347-54.

Yu KD, Liu GY, Di GH, et al (2007). Progesterone receptor status provides predictive value for adjuvant endocrine therapy in older estrogen receptor-positive breast cancer patients. Breast, 16, 307-15. 